\title{
Lower Serum Angiotensin-Converting Enzyme Level in Relation to Hyperinflammation and Impaired Antiviral Immune Response Contributes to Progression of COVID-19 Infection
}

\author{
Yuying Chen - Da Huang - Wei Yuan · Jiang Chang - Zhize Yuan · \\ $\mathrm{Di} \mathrm{Wu} \cdot$ Meifang Han $\cdot$ Xiaoping Luo $\cdot$ Qin Ning $\cdot$ Weiming Yan (B)
}

Received: May 12, 2021 / Accepted: July 26, 2021 / Published online: August 13, 2021

(c) The Author(s) 2021

\begin{abstract}
Introduction: As a homologue of the angiotensin-converting enzyme (ACE), angiotensinconverting enzyme 2 (ACE2) has been identified as the main receptor for severe acute respiratory syndrome coronavirus 2 (SARS-CoV-2) invasion. We aimed to investigate the role of serum ACE in predicting the coronavirus disease 2019 (COVID-19) disease progression and the underlying mechanisms.

Methods: We retrospectively enrolled 120 patients with confirmed COVID-19 who underwent serum ACE detection on admission. The clinical characteristics and laboratory findings during hospitalization were evaluated dynamically to identify the potential risk factors for disease progression.
\end{abstract}

Yuying Chen and Da Huang contributed equally.

Y. Chen · D. Huang $\cdot$ W. Yuan $\cdot$ J. Chang $\cdot$ Z. Yuan

D. Wu $\cdot$ M. Han $\cdot$ Q. Ning $(\varangle) \cdot$ W. Yan $(\bowtie)$

Department and Institute of Infectious Disease,

Tongji Hospital, Tongji Medical College, Huazhong

University of Science and Technology, Wuhan,

China

e-mail: qning@vip.sina.com

W. Yan

e-mail: ywm_net@qq.com

X. Luo

Department of Pediatrics, Tongji Hospital, Tongji Medical College, Huazhong University of Science and Technology, Wuhan, China
Results: ACE level was demonstrated as one of the independent risk factors. Patients with ACE level $\leq 33.5 \mathrm{U} / \mathrm{L}$ showed a higher cumulative virus RNA detection rate, elevated pro-inflammatory mediators levels, declined lymphocyte count, and decreased SARS-CoV-2-specific antibodies than those with ACE level > 33.5 U/L.

Conclusion: Lower serum ACE levels in relation to delayed virus elimination, hyperinflammatory condition, and impaired host antiviral immune responses contribute to disease progression of COVID-19.

Keywords: COVID-19; Serum angiotensinconverting enzyme; Disease progression; Immune response; Virus clearance 


\section{Key Summary Points}

\section{Why carry out this study?}

COVID-19 is still spreading all over the world and risk factors for disease progression warrant further investigation.

As a homologue of the angiotensinconverting enzyme (ACE), angiotensinconverting enzyme 2 (ACE2) has been identified as the main receptor for severe acute respiratory syndrome coronavirus 2 (SARS-CoV-2) invasion.

\section{What was learned from the study?}

Serum ACE level is predictive of COVID-19 disease progression.

Serum ACE level is related to poor clinical outcome, delayed virus clearance, hyperinflammation, and immunosuppression during the course of COVID-19.

\section{INTRODUCTION}

The coronavirus disease 2019 (COVID-19), caused by the novel severe acute respiratory syndrome coronavirus 2 (SARS-CoV-2), is an ongoing global pandemic that has resulted in significant social and economic losses. As reported previously, patients with COVID-19 disease progression had significantly poorer clinical outcome and higher mortality rates than those without [1]. Therefore, identifying risk factors for COVID-19 disease progression has important implications in developing treatment options and rationally allocating medical resources.

Angiotensin-converting enzyme (ACE) is a type 1 transmembrane monomeric glycoprotein that could be classified as a peptidyl dipeptidase belonging to the M2 family of zinc metallopeptidases. ACE is abundant in the lungs, intestine, kidneys, brain, and aorta and is mostly expressed in the luminal membrane of vascular endothelial cells, particularly the pulmonary endothelium [2]. Membrane-bound ACE can be cleaved and transferred into circulation; this process is reportedly regulated by ACE secretase, which has not been identified yet but is believed to be a membrane-bound metalloprotease [3]. ACE plays a key role in the renin-angiotensin system (RAS) by catalyzing conversion of angiotensin I (Ang I) to angiotensin II (Ang II), which then exerts its effects including vasoconstriction, blood pressure elevation, and induction of inflammatory and pro-fibrotic pathways, and vasoconstriction via type 1 receptors (AT1R). The ACE-Ang I-AT1R axis was demonstrated to participate in the progression of acute respiratory distress syndrome (ARDS)/acute lung injury (ALI) [3]. In 2000, angiotensin-converting enzyme 2 (ACE2) was discovered as a close homologue of ACE and could be detected mainly in the intestine, lungs, heart, kidneys, and pancreas $[4,5]$. By acting as an endogenous counter-regulatory axis to the ACE axis, ACE2 converts Ang II into angiotensin 1-7 and exerts vasodilatory, anti-inflammatory, and anti-fibrotic effects via the Mas receptor [2, 4]. Besides being an RAS modulator, ACE2 was identified as the functional receptor for SARS-CoV-2 following the COVID-19 outbreak [6].

It has been indicated in recent years that serum ACE level correlates with the diagnosis and prognosis of a variety of respiratory disorders [7]; however, the potential relationship between ACE level and clinical outcomes of SARS-CoV-2 infection is not adequately understood. The latest research based on 136 patients with confirmed COVID-19 suggested that serum ACE level could serve as a marker to reflect the clinical condition of COVID-19 rather than an independent risk factor for disease severity [8]. In our study, 120 patients with confirmed COVID-19 who underwent measurement of serum ACE levels on admission were retrospectively enrolled, to evaluate the potential role of serum ACE level in combination with other risk factors in predicting disease progression of COVID-19 and further investigate the possible mechanisms involved. 


\section{METHODS}

\section{Participants and Study Design}

Between January 13, 2020 and March 1, 2020, 120 patients with confirmed COVID-19 based on detection of SARS-CoV-2 RNA who were hospitalized at Tongji Hospital of Tongji Medical College, Huazhong University of Science and Technology and underwent detection of serum ACE levels on admission were enrolled in this retrospective study cohort. We collected data on demographics, comorbidities, symptoms and signs, and serum ACE levels and other laboratory findings during the hospitalization from the inpatient electronic medical recording system from Tongji Hospital.

\section{Definitions}

Patients were categorized into the progressive group if they fulfilled any of the following three criteria: use of mechanical ventilation, admission to intensive care unit (ICU), or death; otherwise, they were categorized into the nonprogressive group. Hypoxemia was defined as a $\mathrm{PaO}_{2} / \mathrm{FiO}_{2}$ ratio of less than 300 . Acute kidney injury was diagnosed according to at least one of the three following definitions: (1) increase in serum creatinine (SCr) of $0.3 \mathrm{mg} / \mathrm{dL}$ ( $\geq 26.5 \mu \mathrm{mol} / \mathrm{L}$ ) within $48 \mathrm{~h}$; (2) increase in $\mathrm{SCr}$ to $>1.5$ times the basal value within 7 days of known or assumed renal impairment; (3) urine output $<0.5 \mathrm{~mL} /(\mathrm{kgh})$ for $6 \mathrm{~h}$. Acute liver injury was defined as jaundice with a total bilirubin level of $\geq 3 \mathrm{mg} / \mathrm{dL}$ and an acute increase in alanine transaminase (ALT) by at least five times the upper limit of the normal range and/or an increase in alkaline phosphatase by at least twice the upper limit of the normal range. Cardiac injury was diagnosed if serum levels of cardiac biomarkers (e.g., troponin I) were greater than the 99th percentile of the upper reference limit, or if new abnormalities were detected in electrocardiography and echocardiography.

\section{Detection of SARS-CoV-2 Viral RNA}

Throat or nasopharyngeal swabs were obtained for laboratory testing of SARS-CoV-2. Viral RNA was extracted from clinical samples and further used for real-time reverse transcription polymerase chain reaction (RT-PCR) assay of SARSCoV-2 by using a commercial TaqMan one-step real-time RT-PCR kit from Shanghai BioGerm Medical Biotechnology Co. Ltd. (Shanghai, China). Primers and probes involved were designed to target the open reading frame $1 \mathrm{ab}$ $(\mathrm{ORF} 1 \mathrm{ab})$ and nucleocapsid $(\mathrm{N})$ gene of SARSCoV-2, whose sequences have been described in a previous study [9]. Real-time RT-PCR was performed using the following conditions: $50{ }^{\circ} \mathrm{C}$ for $10 \mathrm{~min}, 95^{\circ} \mathrm{C}$ for $5 \mathrm{~min}$, followed by 40 cycles of $95^{\circ} \mathrm{C}$ for $10 \mathrm{~s}$ and $55^{\circ} \mathrm{C}$ for $40 \mathrm{~s}$. A cycle-count threshold value of $<37$ was defined as positive for SARS-CoV-2 RNA.

\section{Detection of Angiotensin-Converting Enzyme}

ACE activity level in the serum was quantified on the basis of a microplate fluorescent readerbased assay using a commercial ACE assay kit according to the manufacturer's instructions. The ACE Assay Kit (Angiotensin I Converting Enzyme, \#AD309AC) was purchased from Glenbio (UK).

\section{Laboratory Measurements}

Routine laboratory measurements including complete blood count, serum biochemical test (including liver and renal function, creatine kinase, LDH, and electrolytes), blood gas analysis, measurement of serum inflammatory mediators (CRP, ferritin, ESR, and PCT), myocardial enzyme spectrum analysis, and coagulation profile study were performed in the Department of Laboratory Medicine in Tongji Hospital according to the clinical requirement.

Serum cytokine profile was determined by chemiluminescence immunoassay (CLIA) performed on a fully automated analyzer (Siemens Immulite 1000, DiaSorin Liaison, or Roche Diagnostics Cobas e602) according to the 
manufacturers' instructions. CLIA kits for interleukin-1beta (IL-1 $\beta$ ) (LKL11), IL-2R (LKIP1), IL-8 (LK8P1), IL-10 (LKXP1), and tumor necrosis factor alpha (TNF $\alpha)$ (LKNF1) were purchased from DiaSorin. An IL-6 kit $(05,109,442,190)$ was purchased from Roche Diagnostics.

Peripheral lymphocyte subsets, including the proportions and numbers of NK cells, B cells, total $\mathrm{T}$ cells, $\mathrm{CD} 4^{+} \mathrm{T}$ cells, and $\mathrm{CD}^{+} \mathrm{T}$ cells were investigated in all patients. The following reagents were purchased from Becton Dickinson (BD) Biosciences and used according to the manufacturer's instructions: BD Multitest 6-Color TBNK reagent $(\# 644,611)$ and BD Trucount Tubes (\#340,334). BD Multitest 6-Color TBNK reagent contains anti-CD3(SK7, FITC), anti-CD16(B73.1, PE), anti-CD56(NCAM16.2, PE), anti-CD45(2D1, PerCP-Cy5.5), antiCD4(SK3, PE-Cy7), anti-CD19(SJ25C1, APC), and anti-CD8(SK1All, APC-Cy7). All samples were detected by a BD FACS Canto II flow cytometry system and analyzed with the BD FACSDiva software.

Detection of specific antibodies against SARS-CoV-2 was based on an automated paramagnetic particle chemiluminescent immunoassay (CLIA) kit supplied by Yhlo Biotech Co. Ltd. (Shenzhen, China). A test result $\geq 10 \mathrm{AU} / \mathrm{mL}$ was considered reactive (positive).

\section{Statistics}

Continuous variables were expressed as mean and standard error of the mean (SEM) and compared with the unpaired two-sided Student's $t$ test; categorical variables were expressed as number (\%) and compared using the chisquared test or Fisher's exact test between the two study groups. Prediction value was appraised by the receiver operating characteristic (ROC) curves. The 95\% confidence interval (CI) was utilized to calculate the sensitivity and specificity; the cutoff value was selected when the Jordan index was at its maximum. Kaplan-Meier analysis was conducted to value the survival rate and cumulative RNA positive rate. Statistical analyses were carried out using SPSS (version 19.0, IBM). a two-sided
$P$ value $<0.05$ was considered to indicate statistical significance.

\section{Study Approval}

The study was performed in accordance with the tenets of the Declaration of Helsinki and the Good Clinical Practice principles. The case series was approved by the Institutional Review Board of Tongji Hospital of Tongji Medical College, Huazhong University of Science and Technology (TJ-C20200101). Written informed consent was waived because of the rapid emergence of this infectious disease.

\section{RESULTS}

\section{Baseline Demographics and Disease Characteristics}

As summarized in Table 1, the cohort comprised 120 enrolled patients: 24 showed disease progression during hospitalization (progressive group) whereas the remaining 96 patients did not (non-progressive group). The distribution of sex between the two groups was comparable, while the mean age of the progressive group was significantly higher than that of the non-progressive group (63.7 vs. 56.8 years, $P=0.034$ ). Patients in the progressive group were more likely to have at least one underlying comorbidity $(62.5 \%$ vs. $37.5 \%, P=0.037)$, especially a possible comorbidity of diabetes $(45.8 \%$ vs. $14.6 \%, P=0.002$ ) than those in the non-progressive group. Although serum level of ACE has seldom been discussed as a risk factor for COVID-19 disease progression in the existing literature, significantly lower ACE levels were found in patients in the progressive than nonprogressive group (mean 18.5 vs. $28.0 \mathrm{U} / \mathrm{L}$, $P=0.002$ ), suggesting its potential use as a biomarker of disease progression. Much more frequent fever and higher peak temperature were found in the progressive than non-progressive group $[23(95.8 \%)$ vs. $74(77.1 \%)$, $P=0.042$ and $39.0^{\circ} \mathrm{C}$ vs. $\left.38.1^{\circ} \mathrm{C}, P=0.000\right]$. Dyspnea was significantly more frequent in the progressive group than in the non-progressive 
Table 1 Baseline demographics and disease characteristics

$\begin{array}{llll}\begin{array}{l}\text { All patients } \\ (n=120)\end{array} & \begin{array}{l}\text { Progression } \\ (n=24)\end{array} & \begin{array}{l}\text { Non-progression } \\ (n=96)\end{array} & P \text { value }\end{array}$

Characteristics

Male, $n(\%)$

$$
56(46.7 \%)
$$

Age, years

Any comorbidity, $n$ (\%)

Hypertension, $n(\%)$

Diabetes, $n(\%)$

ACE, U/L

Signs and symptoms

Fever, $n(\%)$

Highest temperature, ${ }^{\circ} \mathrm{C}$

Cough, $n(\%)$

Sputum production, $n(\%)$

Chest tightness, $n$ (\%)

Dyspnea, $n$ (\%)

Fatigue, $n(\%)$

Diarrhea, $n(\%)$

Myalgia, $n$ (\%)

Headache, $n$ (\%)

Heart rate, bpm

Respiratory rate, per min

$\geq 30, n(\%)$

Percutaneous oxygen saturation

$\leq 93 \%, n / N(\%)$

$\mathrm{PaO}_{2} / \mathrm{FiO}_{2}, \mathrm{mmHg}$

$<300 \mathrm{mmHg}, n / N(\%)$

Laboratory examinations

White blood cell count, $\times 10^{9} / \mathrm{L}$

$>10, n(\%)$

Neutrophil count, $\times 10^{9} / \mathrm{L}$

$>6.3, n(\%)$

Lymphocyte count, $\times 10^{9} / \mathrm{L}$

$<1, n(\%)$

$$
58.1(1.3)
$$

$51(42.5 \%)$

$35(29.2 \%)$

$25(20.8 \%)$

$26.1(1.4)$

$97(80.8 \%)$

$38.3(0.1)$

$94(78.3 \%)$

$54(45.0 \%)$

$62(51.7 \%)$

$44(36.7 \%)$

$42(35.0 \%)$

$32(26.7 \%)$

$24(20.0 \%)$

$15(12.5 \%)$

$92.3(1.4)$

$21.6(0.5)$

$9(7.5 \%)$

$94.9(0.6)$

30/118 (25.4\%)

234.3 (20.9)

$14 / 20(70.0 \%)$

$6.5(0.3)$

$9(7.5 \%)$

$4.7(0.3)$

$16(13.3 \%)$

$1.2(0.1)$

$58(48.3 \%)$
$11(45.8 \%)$

63.7 (2.6)

$15(62.5 \%)$

$9(37.5 \%)$

$11(45.8 \%)$

$18.5(1.7)$

$23(95.8 \%)$

$39.0(0.2)$

$20(83.3 \%)$

$12(50.0 \%)$

$15(62.5 \%)$

$16(66.7 \%)$

$12(50.0 \%)$

$8(33.3 \%)$

$8(33.3 \%)$

$4(16.7 \%)$

91.4 (3.2)

24.9 (2.0)

$4(16.7 \%)$

$90.4(2.1)$

10/23 (43.5\%)

$169.0(27.2)$

$6 / 6(100.0 \%)$

8.9 (1.0)

7 (29.2\%)

$7.7(0.9)$

$12(50.0 \%)$

$0.7(0.1)$

$19(79.2 \%)$

$$
45(46.9 \%)
$$

1

56.8 (1.5)

$0.034^{*}$

$36(37.5 \%)$

$0.037^{*}$

$26(27.1 \%)$

0.325

$14(14.6 \%)$

$0.002^{*}$

$28.0(1.6)$

$0.002^{*}$

$$
74(77.1 \%) \quad 0.042^{*}
$$

$38.1(0.1)$

$0.000^{*}$

$74(77.1 \%)$

0.591

$42(43.8 \%)$

0.65

$47(49.0 \%)$

0.261

$28(29.2 \%)$

$0.002^{*}$

$30(31.3 \%)$

0.098

$24(25.0 \%)$

0.444

$16(16.7 \%)$

0.087

$11(11.5 \%)$

0.497

$92.5(1.6)$

0.76

$20.8(0.4)$

$0.001^{*}$

$5(5.2 \%)$

0.078

$96.0(0.5)$

$0.002^{*}$

20/95 (21.1\%) $0.035^{*}$

$262.3(24.2) \quad 0.036^{*}$

$8 / 14(57.1 \%) \quad 0.115$ 
Table 1 continued

\begin{tabular}{|c|c|c|c|c|}
\hline & $\begin{array}{l}\text { All patients } \\
(n=120)\end{array}$ & $\begin{array}{l}\text { Progression } \\
(n=24)\end{array}$ & $\begin{array}{l}\text { Non-progression } \\
(n=96)\end{array}$ & $P$ value \\
\hline Platelet count, $\times 10^{9} / \mathrm{L}$ & $229.6(7.6)$ & $231.9(19.8)$ & $229.0(8.2)$ & 0.882 \\
\hline $\mathrm{ALT}, \mathrm{U} / \mathrm{L}$ & $36.9(4.5)$ & $57.1(16.1)$ & $31.8(3.8)$ & 0.062 \\
\hline AST, U/L & $32.8(1.9)$ & $44.4(4.8)$ & $29.9(2.0)$ & $0.003^{*}$ \\
\hline Albumin, $\mathrm{g} / \mathrm{L}$ & $35.7(0.5)$ & $30.7(0.8)$ & $36.9(0.6)$ & $<0.001^{*}$ \\
\hline Total bilirubin, $\mu \mathrm{mol} / \mathrm{L}$ & $10.0(0.9)$ & $15.0(4.0)$ & $8.8(0.4)$ & $0.003^{*}$ \\
\hline Creatine kinase, $\mathrm{U} / \mathrm{L}$ & $93.9(17.3)$ & $177.7(84.1)$ & $74.6(7.8)$ & 0.312 \\
\hline Lactate dehydrogenase, $\mathrm{U} / \mathrm{L}$ & $304.6(13.8)$ & $452.9(40.6)$ & $267.5(11.1)$ & $<0.001^{*}$ \\
\hline Blood urea nitrogen, $\mathrm{mmol} / \mathrm{L}$ & $6.6(1.6)$ & $8.2(1.6)$ & $6.3(1.9)$ & $0.002^{*}$ \\
\hline Creatinine, $\mu \mathrm{mol} / \mathrm{L}$ & $73.4(3.5)$ & $88.7(15.7)$ & $69.5(1.8)$ & 0.88 \\
\hline eGFR, $\mathrm{mL} /\left(\min 1.73 \mathrm{~m}^{2}\right)$ & $92.0(1.8)$ & $85.8(5.9)$ & $93.6(1.7)$ & 0.337 \\
\hline Troponin, $\mathrm{pg} / \mathrm{mL}$ & $243.5(164.5)$ & $519.3(485.4)$ & $172.3(165.7)$ & $<0.001^{*}$ \\
\hline $\mathrm{BNP}, \mathrm{pg} / \mathrm{mL}$ & $919.6(613.0)$ & $3766.0(3014.0)$ & $200.1(34.0)$ & $<0.001^{*}$ \\
\hline Prothrombin time, $s$ & $14.6(0.6)$ & $14.7(0.2)$ & $14.6(0.7)$ & 0.987 \\
\hline $\mathrm{D}$-Dimer, $\mu \mathrm{g} / \mathrm{mL}$ & $2.6(0.5)$ & $6.8(1.6)$ & $1.6(0.4)$ & $<0.001^{*}$ \\
\hline Procalcitonin, $\mathrm{ng} / \mathrm{mL}$ & $0.14(0.02)$ & $0.27(0.05)$ & $0.10(0.03)$ & $0.004^{*}$ \\
\hline C-reactive protein, $\mathrm{mg} / \mathrm{L}$ & $48.4(5.9)$ & $110.0(19.0)$ & $34.3(4.8)$ & $<0.001^{*}$ \\
\hline Ferritin, $\mu \mathrm{g} / \mathrm{L}$ & $1078(245.9)$ & $1951.0(520.8)$ & $532.1(159.7)$ & $0.000^{*}$ \\
\hline $\mathrm{ESR}, \mathrm{mm} / \mathrm{h}$ & $37.8(3.5)$ & $52.1(6.3)$ & $32.2(4.0)$ & $0.009^{*}$ \\
\hline IL- $1 \beta, \mathrm{pg} / \mathrm{mL}$ & $6.0(0.2)$ & $5.3(0.2)$ & $6.2(0.3)$ & 0.262 \\
\hline $\mathrm{IL}-2 \mathrm{R}, \mathrm{U} / \mathrm{mL}$ & $722(46.3)$ & $1181.0(123.4)$ & $602.2(40.6)$ & $<0.001^{*}$ \\
\hline IL-6, pg/mL & $17.2(3.5)$ & $47.7(14.4)$ & $9.3(1.5)$ & $<0.001^{*}$ \\
\hline $\mathrm{IL}-8, \mathrm{pg} / \mathrm{mL}$ & $19.3(2.5)$ & $28.9(5.1)$ & $16.8(2.8)$ & 0 \\
\hline IL-10, pg/mL & $6.4(0.4)$ & $9.2(1.4)$ & $5.7(0.2)$ & $<0.001^{*}$ \\
\hline $\mathrm{TNF} \alpha, \mathrm{pg} / \mathrm{mL}$ & $9.9(0.7)$ & $12.7(2.2)$ & $9.2(0.7)$ & $0.047^{*}$ \\
\hline
\end{tabular}

Data are expressed as mean (SEM), $n(\%)$, or $n / N(\%)$, where $N$ is the total number of patients with available data. $P$ values comparing progression and non-progression are from $\chi^{2}$ test, Fisher's exact test, unpaired two-sided Student's $t$ test or Mann-Whitney $U$ test

group [16 (66.7\%) vs. $28(29.2 \%), P=0.002]$. The frequencies of other prevalent symptoms at onset of illness including cough, sputum production, and chest tightness, and frequencies of less common symptoms including diarrhea, myalgia, and headache were comparable between the two groups. According to vital signs recorded on the day of hospital admission, significantly higher respiratory rate and lower oxygen saturation were found in the progressive patients than the non-progressive patients (mean $24.9 / \mathrm{min}$ vs. $20.8 / \mathrm{min}, P=0.001$; and 
mean $90.4 \%$ vs. $96.0 \%, P=0.002)$. Further, $43.5 \%(10 / 23)$ of progressive patients but only $21.1 \%(20 / 95)$ of non-progressive patients had percutaneous oxygen saturation of $\leq 93.0 \%$ on admission $(P=0.035)$.

Among the 20 patients who underwent arterial blood gas (ABG) testing, the $\mathrm{PaO}_{2} / \mathrm{FiO}_{2}$ ratio was obviously lower in the progressive than non-progressive patients (mean 169.0 vs. $262.3 \mathrm{mmHg}, P=0.036$ ). Moreover, all six progressive cases showed a $\mathrm{PaO}_{2} / \mathrm{FiO}_{2}$ ratio $<300 \mathrm{mmHg}$, indicating the occurrence of acute respiratory distress syndrome in these patients.

Laboratory findings showed remarkably higher counts of white blood cells (WBCs) and neutrophils; higher levels of aspartate aminotransferase (AST), total bilirubin (TBiL), lactate dehydrogenase (LDH), blood urea nitrogen (BUN), troponin, BNP, D-dimer, procalcitonin (PCT), C-reactive protein (CRP), ferritin, erythrocyte sedimentation rate (ESR), IL-2R, IL-6, IL-8, IL-10, and TNF $\alpha$; but lower lymphocyte count albumin level in the progressive than non-progressive patients. More patients with progressive disease developed lymphopenia and leukocytosis than those with non-progressive disease $[19 / 24 \quad(79.2 \%)$ vs. $39 / 96 \quad(40.6 \%)$, $P=0.001 ; \quad 7 / 24 \quad(29.2 \%) \quad$ vs. $2 / 96 \quad(2.1 \%)$, $P<0.001]$. These findings suggest that impairment of multiple organ function and systemic inflammation are more prevalent among patients with COVID-19 disease progression.

\section{Serum ACE Level is Predictive of COVID- 19 Disease Progression}

In the univariate analysis, ACE level, age, underlying diabetes, and lymphocyte count on admission were found to correlate with COVID19 disease progression. Further multivariate logistic analysis indicated that ACE level, underlying diabetes, and lymphocyte count on admission were independent risk factors for COVID-19 disease progression (Table 2). As for their performance for predicting disease progression, the ROC curves displayed the area under the curve (AUC) of serum ACE, underlying diabetes, and lymphocyte count as 0.701 $(95 \% \quad$ CI $\quad 0.594-0.809), \quad 0.651 \quad(95 \% \quad$ CI 0.518-0.784), and 0.794 (95\% CI 0.705-0.883), respectively. Combination of serum ACE level and underlying diabetes or lymphocyte count exhibited a superior performance for predicting COVID-19 disease progression than any single predictor, with an increased AUC of 0.829 (95\% CI $0.745-0.912)$ and $0.857 \quad(95 \% \quad$ CI 0.777-0.936), respectively. A composite predictor consisting of ACE level, underlying diabetes, and lymphocyte count further improved the predictive abilities of coupled predictors, although the increase in AUC was modest (0.860 [95\% CI 0.786-0.933]) (Fig. 1a). We determined an optimal ACE cutoff value of $33.5 \mathrm{U} / \mathrm{L}$ to predict disease progression with a sensitivity specificity, positive predictive value, and negative predictive value of $100 \%, 30.2 \%$, $26.4 \%$, and $100 \%$, respectively (Fig. 1b). These

Table 2 Univariate analysis and multivariate analysis of disease progression risk factors

\begin{tabular}{|c|c|c|c|c|}
\hline & \multicolumn{2}{|c|}{ Univariate analysis } & \multicolumn{2}{|c|}{ Multivariate analysis } \\
\hline & $P$ value & OR $(95 \% \mathrm{CI})$ & $P$ value & OR $(95 \% \mathrm{CI})$ \\
\hline $\mathrm{ACE}(\mathrm{U} / \mathrm{L})$ & 0.004 & $0.931(0.887-0.978)$ & 0.008 & $0.929(0.880-0.981)$ \\
\hline Age (year) & 0.038 & $1.040(1.002-1.079)$ & & \\
\hline Diabetes (yes vs. no) & 0.002 & $4.569(1.726-12.099)$ & 0.009 & $5.266(1.501-18.475)$ \\
\hline Lymphocyte count $\left(\times 10^{9} / \mathrm{L}\right)$ & 0 & $0.079(0.021-0.292)$ & 0.001 & $0.075(0.016-0.343)$ \\
\hline
\end{tabular}



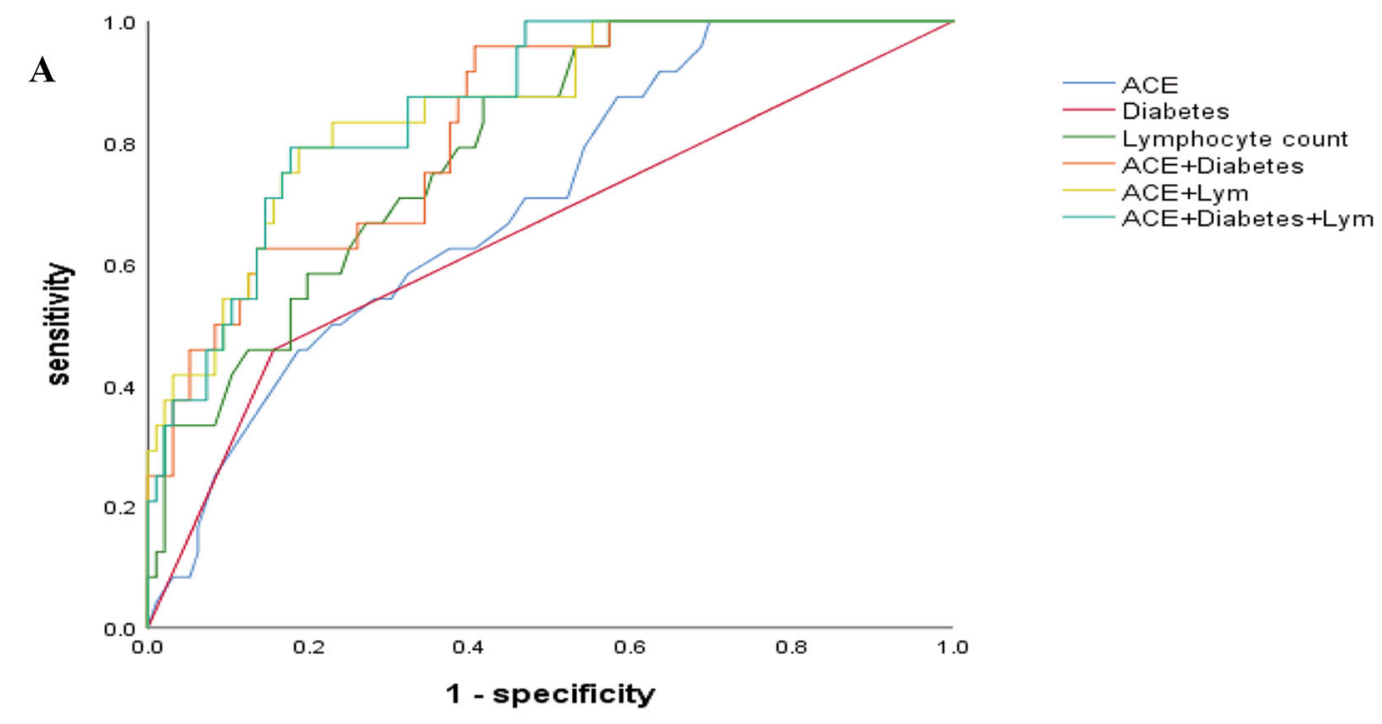

\begin{tabular}{ccc}
\hline & AUC $(95 \% \mathrm{Cl})$ & P value \\
\hline ACE & $0.701(0.594-0.809)$ & 0.002 \\
Diabetes & $0.651(0.518-0.784)$ & 0.022 \\
Lymphocyte count & $0.794(0.705-0.883)$ & 0.000 \\
ACE+Diabetes & $0.829(0.745-0.912)$ & 0.000 \\
ACE+Lym & $0.857(0.777-0.936)$ & 0.000 \\
ACE+Diabetes+Lym & $0.860(0.786-0.933)$ & 0.000 \\
\hline
\end{tabular}

\section{B}

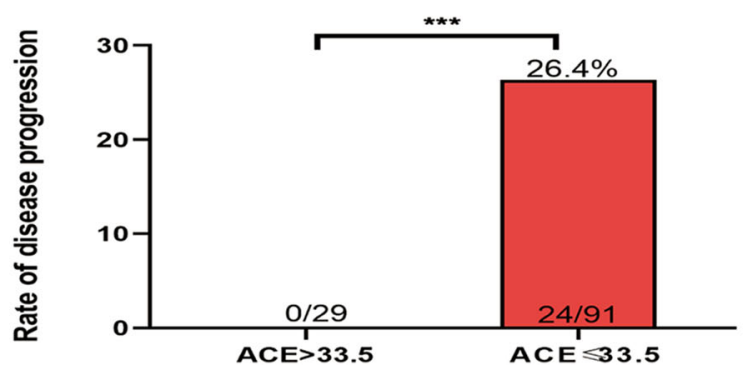

Fig. 1 Serum ACE level, diabetes, and lymphocyte count on admission are predictors of COVID-19 disease progression. a ROC curve analysis of ACE, diabetes, lymphocyte count, and their combination in patients with COVID-19 to predict the disease progression. b Optimal

results implied the relationship between serum ACE level and COVID-19 prognosis, and indicated the potential contribution of ACE level to the development of a predictive model for COVID-19 disease progression.
ACE cutoff value to distinguish disease progression cases from non-progressive cases. ACE angiotensin-converting enzyme, ROC receiver operating characteristic. ${ }^{* * *} P<0.0001,{ }^{* * *} P<0.001,{ }^{* *} P<0.01$, and ${ }^{*} P<0.05$

\section{Serum ACE Level in Relation to Poor Clinical Outcome}

We further compared the baseline characteristics and clinical outcomes between patients 
Table 3 Baseline characteristics and clinical outcomes of patients with COVID-19 between ACE $\leq 33.5$ and ACE $>33.5$

\begin{tabular}{lcccc}
\hline & $\begin{array}{l}\text { All patients } \\
(\boldsymbol{n}=\mathbf{1 2 0})\end{array}$ & $\begin{array}{l}\text { ACE } \leq 33.5 \\
(\boldsymbol{n}=\mathbf{9 1})\end{array}$ & $\begin{array}{l}\text { ACE }>33.5 \\
(\boldsymbol{n}=\mathbf{2 9})\end{array}$ & $\boldsymbol{P}$ value \\
\hline Characteristics & $56(46.7 \%)$ & $41(45.1 \%)$ & $15(51.7 \%)$ & 0.67 \\
Male, $n$ (\%) & $58.1(55.6-60.7)$ & $58.9(56.3-61.7)$ & $55.6(48.8-62.4)$ & 0.499 \\
Age, years & $51(42.5 \%)$ & $41(45.1 \%)$ & $10(34.5 \%)$ & 0.39 \\
Any comorbidity, $n(\%)$ & $35(29.2 \%)$ & $28(30.8 \%)$ & $7(24.1 \%)$ & 0.64 \\
Hypertension, $n$ (\%) & $25(20.8 \%)$ & $19(20.9 \%)$ & $6(20.7 \%)$ & 1 \\
Diabetes, $n$ (\%) & $23(19.2 \%)$ & $23(25.3 \%)$ & $0(0.0 \%)$ & $0.001^{*}$ \\
Clinical outcomes & $8 / 117(6.8 \%)$ & $8 / 88(9.1 \%)$ & $0 / 29(0 \%)$ & 0.093 \\
Mechanical ventilation, $n$ & $33(27.5 \%)$ & $31(34.1 \%)$ & $2(6.9 \%)$ & $0.004^{*}$ \\
Death, $n / N$ & $9 / 116(7.8 \%)$ & $9 / 87(10.3 \%)$ & $0 / 29(0.0 \%)$ & 0.071 \\
Any complication, $n(\%)$ & $10 / 115(8.7 \%)$ & $10 / 87(11.5 \%)$ & $0 / 28(0.0 \%)$ & 0.061 \\
ARDS, $n / N$ & $22 / 117(18.8 \%)$ & $20 / 89(22.5 \%)$ & $2 / 28(7.1 \%)$ & 0.07 \\
Acute cardiac injury, $n / N$ & $10 / 118(8.5 \%)$ & $10 / 89(11.2 \%)$ & $0 / 29(0.0 \%)$ & 0.059 \\
Acute kidney injury, $n / N$ & & & & \\
Acute liver injury, $n / N$ & & & & \\
\hline
\end{tabular}

Data are median (IQR), $n$ (\%), or $n / N(\%)$, where $N$ is the total number of patients with available data. $P$ values comparing severe cases and moderate cases are from $\chi^{2}$ test, Fisher's exact test, unpaired two-sided Student's $t$ test or Mann-Whitney $U$ test

$A R D S$ acute respiratory distress syndrome

with COVID-19 with an ACE $\leq 33.5 \mathrm{U} / \mathrm{L}$ and those with an ACE $>33.5 \mathrm{U} / \mathrm{L}$. As shown in Table 3, there was no significant difference in the relative distributions of sex, age, and comorbidities between the two groups. However, a significantly increased requirement of mechanical ventilation and higher incidence of complications including ARDS, acute kidney injury, acute cardiac injury, and acute liver injury were found in patients with $\mathrm{ACE} \leq 33.5 \mathrm{U} / \mathrm{L}$ than in those with ACE $>33.5 \mathrm{U} / \mathrm{L}$. Moreover, it is worth noting that all eight deaths occurred in those with ACE $\leq 33.5 \mathrm{U} / \mathrm{L}$, whereas none of the patients with ACE $>33.5 \mathrm{U} / \mathrm{L}$ developed ARDS, acute kidney injury, acute liver injury, or were in need of mechanical ventilation. This provided us with an explanation for why lower ACE level could act as a predictor of disease progression in SARS-CoV-2 infection.

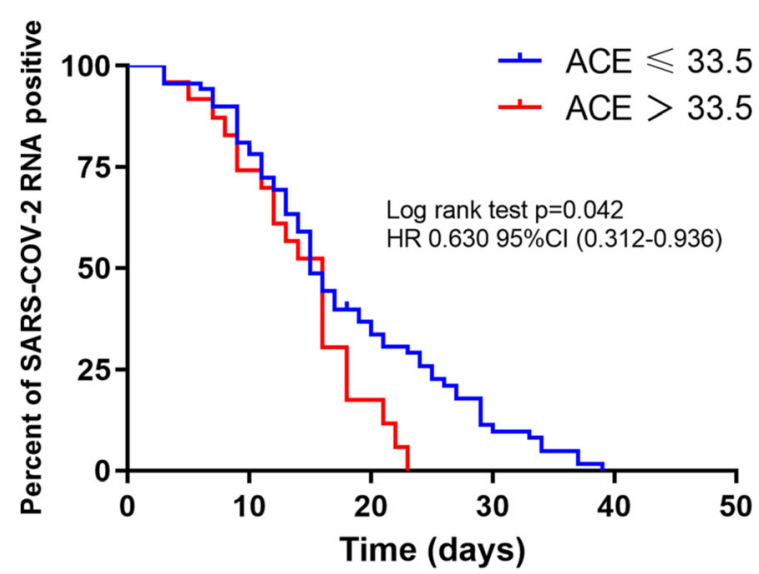

Fig. 2 Kaplan-Meier survival curve for statistical analysis. Patients with $\mathrm{ACE} \leq 33.5 \mathrm{U} / \mathrm{L}$ showed a higher cumulative detection rate of positive viral RNA $(33.74 \%$ vs. $17.46 \%, 9.71 \%$ vs. $0 \%$ at days 20 and 30 post illness onset, respectively) 


\section{Serum ACE Level in Relation to Delayed Virus Clearance}

The cumulative detection rate of positive viral RNA was also evaluated in patients with different levels of ACE. As shown in Fig. 2, when compared to patients with an ACE $>33.5 \mathrm{U} / \mathrm{L}$, those with ACE $\leq 33.5 \mathrm{U} / \mathrm{L}$ exhibited a significantly elevated cumulative detection rate of positive viral RNA during hospitalization (33.7\% vs. $17.5 \%, 9.7 \%$ vs. $0.0 \%$ at days 20 and 30 post illness onset, respectively), suggesting that ACE might participate in the prognosis of COVID-19 by affecting viral invasion and replication.

\section{Serum ACE Level in Relation to Hyperinflammation and Immunosuppression During the Course of COVID-19}

To further explore the possible mechanisms underlying the effect of ACE on COVID-19 disease progression, variations in the parameters reflecting inflammation and immune status were evaluated in patients with various levels of serum ACE. Overproduction of proinflammatory cytokines has been demonstrated as one of the most important factors in determining the severe deterioration of COVID-19. Among available key cytokines observed in this study, larger amounts of IL-2R, IL-6, and IL-10 were observed in patients who had an ACE $\leq 33.5 \mathrm{U} /$ L than in those with ACE $>33.5 \mathrm{U} / \mathrm{L}$ during the first 3 weeks of hospitalization, with the most striking change being concentrated within 7 days after admission (Fig. 3a). Inflammatory mediators including CRP, ferritin, and ESR in patients with $\mathrm{ACE} \leq 33.5 \mathrm{U} / \mathrm{L}$ were markedly higher than those in patients with ACE $>33.5 \mathrm{U} / \mathrm{L}$, with the most striking difference detected on admission and day 21 for CRP, on day 7 for ESR, and on admission for ferritin. Serum levels of PCT were comparable between the two groups (Fig. 3b). We next analyzed the dynamic changes in the peripheral lymphocyte count during hospitalization, lymphocyte subsets, and specific antibody responses against SARS-CoV-2 in a fraction of patients who
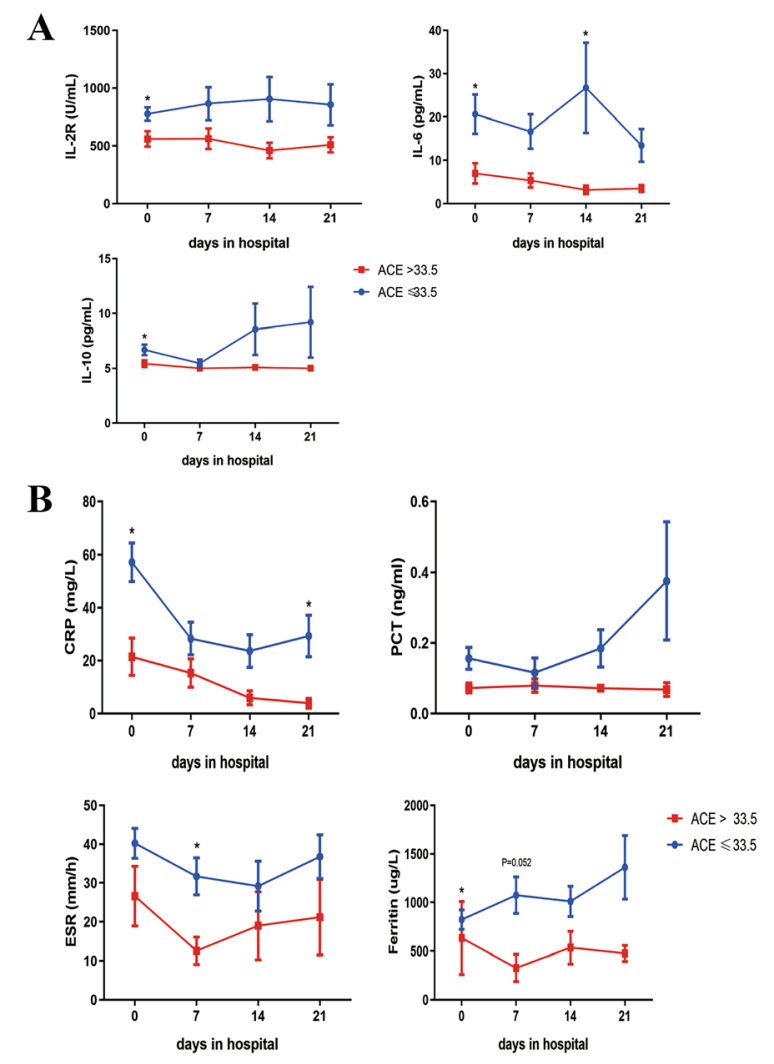

Fig. 3 Comparisons of the proinflammatory cytokines and inflammatory mediators between patients with $\mathrm{ACE} \leq 33.5 \mathrm{U} / \mathrm{L} \quad(n=91)$ and patients with ACE $>33.5 \mathrm{U} / \mathrm{L}(n=29)$ during hospitalization. The comparison of IL-2R, IL-6, IL-10 (a); CRP, PCT, ESR, and ferritin (b) between patients with ACE $\leq 33.5 \mathrm{U} / \mathrm{L}$ and patients with $\mathrm{ACE}>33.5 \mathrm{U} / \mathrm{L}$ on admission and days 7,14 , and 21

underwent two consecutive detections with an interval of $7 \pm 3$ days. As shown in Fig. $4 \mathrm{a}$, patients with ACE $\leq 33.5 \mathrm{U} / \mathrm{L}$ had a significantly lower lymphocyte count on admission, day 7, and day 28 than those with ACE $>33.5 \mathrm{U} / \mathrm{L}$. With regard to peripheral lymphocyte subsets compared between the two detections, there were no statistically significant differences in the absolute numbers of lymphocyte subsets for patients with ACE $>33.5 \mathrm{U} /$ L. In contrast, patients with $\mathrm{ACE} \leq 33.5 \mathrm{U} / \mathrm{L}$ were characterized by a significant decline in total T, CD4 $4^{+} \mathrm{T}, \mathrm{B}$, and NK cell counts, and their NK cell counts during the second testing were notably lower than that of patients with ACE $>33.5 \mathrm{U} / \mathrm{L}$. Our results also indicated that 

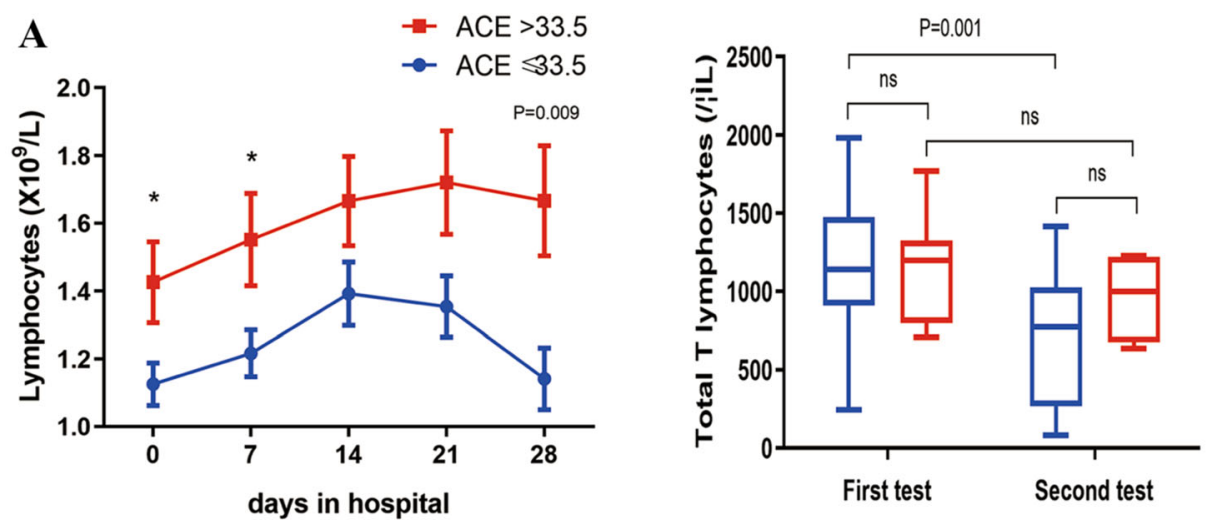

ACE $\$ 33.5$

$\square$ ACE $>33.5$
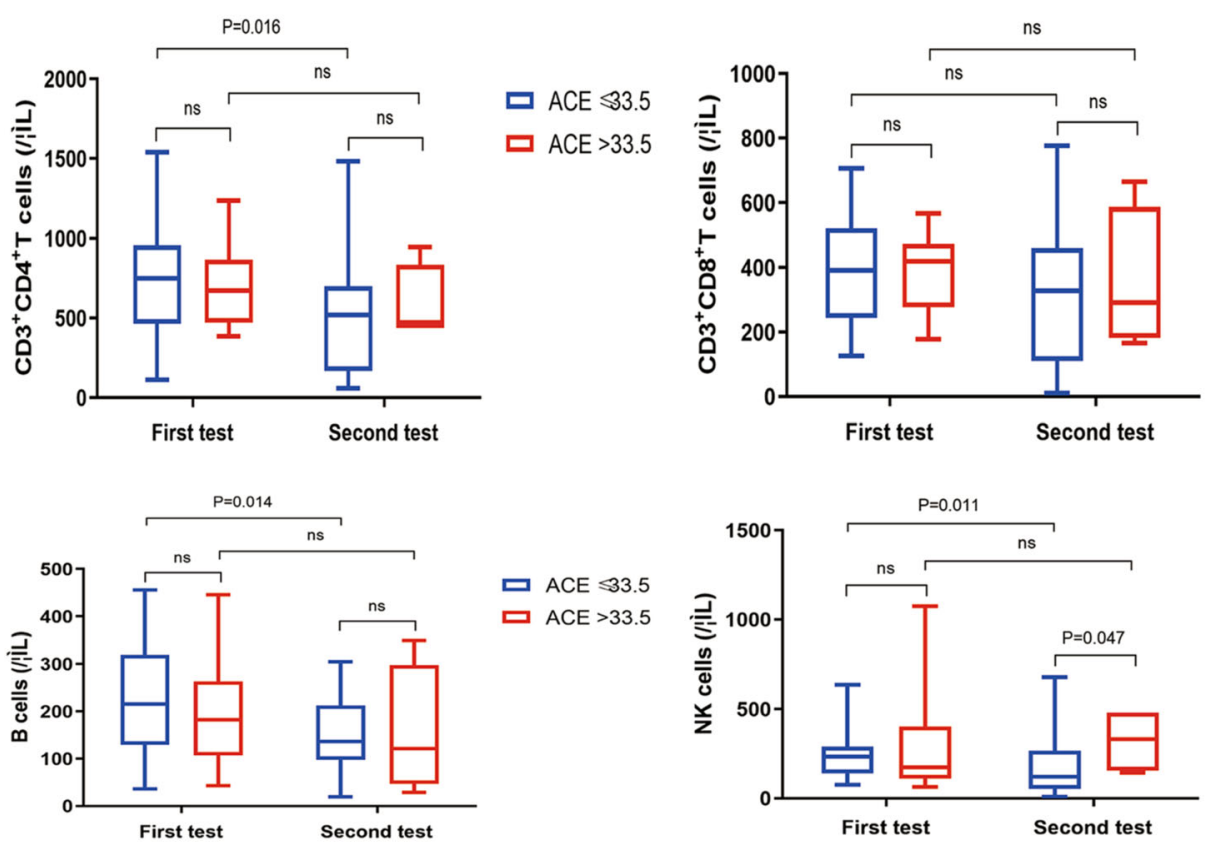

口 ACE $\$ 3.5$

ㅁ $\mathrm{ACE}>33.5$

口 ACE $\$ 3.5$

ㅁ $\mathrm{ACE}>33.5$
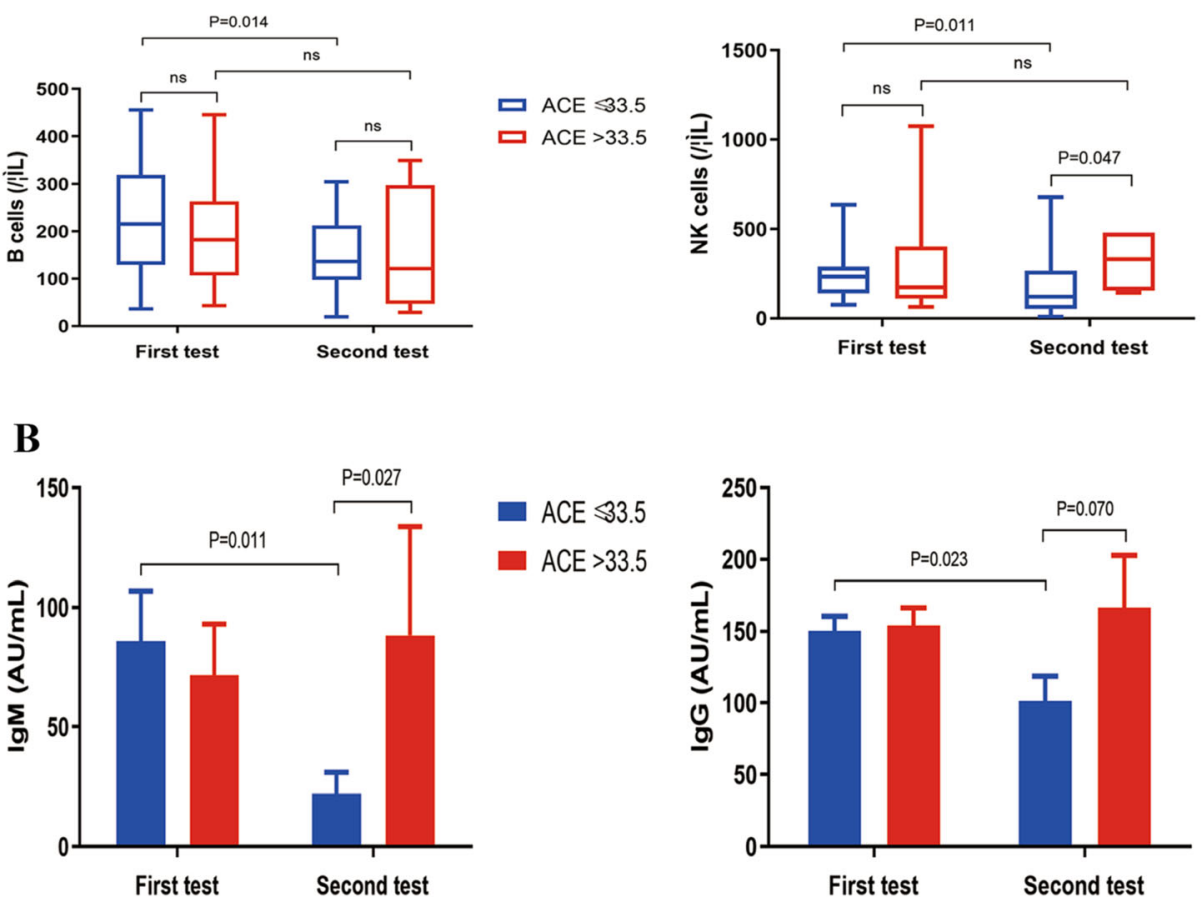

口 $\mathrm{ACE} \$ 33.5$ 口 ACE $>33.5$

First test

口 $\mathrm{ACE} \$ 33.5$

ㅁ $\mathrm{ACE}>33.5$

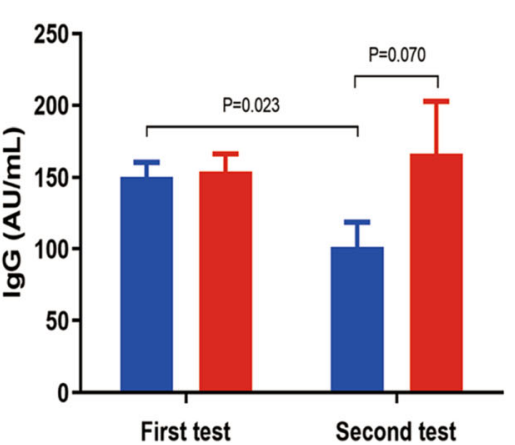

ACE $\$ 33.5$

ACE $>33.5$

ACE $>33.5$

Fig. 4 Comparisons of the immune status between patients. The comparison of lymphocyte count in patients during hospitalization. The change in total $\mathrm{T}$ lymphocytes and $\mathrm{CD}^{+} \mathrm{T}, \mathrm{CD}^{+} \mathrm{T}, \mathrm{B}$, and $\mathrm{NK}$ cells (a), specific

antibody IgM, and IgG against SARS-CoV-2 (b) between two consecutive detections with an interval of $7 \pm 3$ days in patients. ${ }^{* * *} P<0.0001,{ }^{* * *} P<0.001,{ }^{* *} P<0.01$, and ${ }^{*} P<0.05$ 
both IgM and IgG titers were significantly reduced in patients with $\mathrm{ACE} \leq 33.5 \mathrm{U} / \mathrm{L}$, and these patients also displayed dramatically less production of specific IgM antibody as well as a slightly lower level of IgG according to the results of their second round of testing than those with ACE $>33.5$ U/L (Fig. 4b). These laboratory findings were indicative of a relatively hyperinflammatory but immunosuppressive status in patients with lower ACE levels than those with higher ACE levels.

\section{DISCUSSION}

In this real-world retrospective cohort of 120 patients with confirmed SARS-CoV-2 infection, comparison of the clinical and laboratory findings between patients with and without disease progression revealed that lower ACE level on admission correlated with higher incidence of disease progression. To our knowledge, this is the first study to display the potential role of serum ACE as an independent risk factor for COVID-19 disease progression, which might be attributed to multiple mechanisms including delayed elimination of virus, hyperinflammation, and impairment of host immune response (Fig. 5).
At the time of generating this report (May 11, 2020), SARS-CoV-2 infection was a recognized global pandemic, threatening the health of more than 150 million human beings [10]. There is an urgent need to improve our understanding of the pathogenesis, risk factors, and clinical management of this novel disease. Currently, risk factors including older age, underlying comorbidities, lymphopenia, and cytokine storm have been suggested to contribute to COVID-19 disease progression $[1,11,12]$. A retrospective, multicenter, Chinese study of 150 patients with confirmed SARSCoV-2 infection has shown that patients who died from COVID-19 had significantly lower lymphocyte counts than survivors, and elevated ferritin, ESR, and IL- 6 could be used as predictors of fatality [13]. In accordance with these findings, an older age predominance, more frequent occurrence of underlying comorbidities, high fever, low oxygen saturation, impairment of multiple organ function, and systemic inflammation on admission were evidenced in the progressive cases in our study. At present, there are only few studies on the role of ACE in SARS-CoV-2 infection. In a recently published research article, significantly decreased baseline serum ACE level was observed in patients with COVID-19, which gradually increased to the

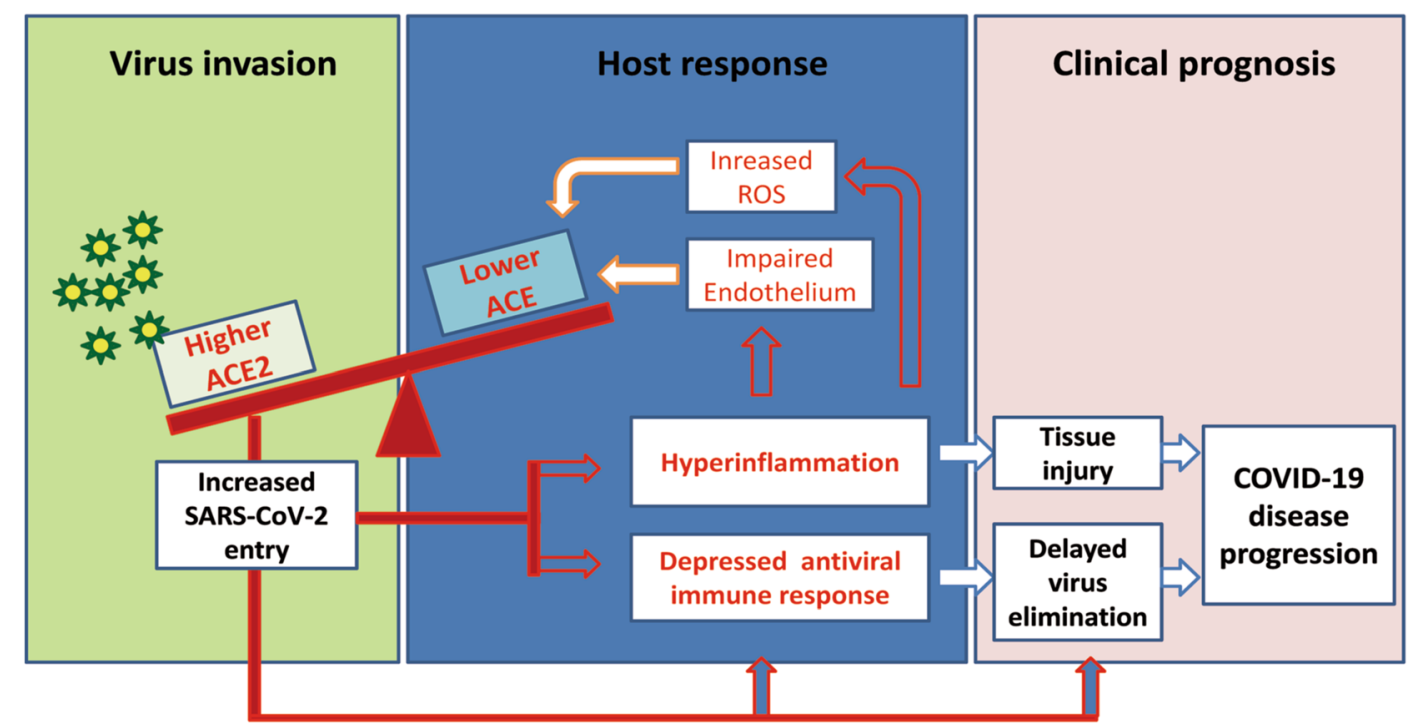

Fig. 5 Schematic outline of low serum ACE level in relation to inflammation, immune status, and clinical outcome 
normal level with disease remission. In that study, although the lowest ACE level was noted in the group with more severe infection, baseline ACE failed to serve as an independent risk factor for disease severity of COVID-19 [8]. To the best of our knowledge, this is the first investigation into whether, and to what extent, a relationship exists between ACE level and COVID-19 disease progression. Our data suggests that lower ACE levels on admission, observed in the progressive cases, might be an efficient predictor and independent risk factor of COVID-19 disease progression, and the predictive performance of which would be improved when combined with underlying diabetes and decreased lymphocyte count.

Since ACE2 has been demonstrated as the SARS-CoV-2 receptor, the scientific community rejected concerns about whether to continue the administration of ACE inhibitor (ACEI) and angiotensin receptor blockers (ARBs) in patients with cardiovascular disease (CVD) complicated with COVID-19. Although many researchers discourage the discontinuation of ACEI and ARBs [14], controversies still exist. A Korean clinical trial concluded that ACEI or ARBs treatment in severe patients with COVID-19 was correlated with the incidence of complications and increased in-hospital mortality. Patients who received higher dose of ACEI or ARBs may experience stronger potentially harmful adverse effects [15]. Additionally, administration of ACEI was reported to potentially decrease serum ACE level [16], which was in favor of our finding that lower serum ACE level was related to worse outcome in patients infected with SARS-CoV-2.

Changes in serum ACE have been previously investigated in multiple respiratory diseases including intrinsic and extrinsic asthma, squamous cell carcinoma of the lung, small cell carcinoma of the lung, sarcoidosis, and emphysema [7, 17]. Despite failing to confirm the prognostic value of serum ACE level regarding clinical outcome in patients with community-acquired pneumonia, researchers from the Netherlands showed a significantly decreased serum ACE level during the acute phase of pneumonia, and further indicated high incidence of bacteremia in patients with low serum ACE level at the time of hospital admission [3]. In line with the aforementioned findings, we found that patients with COVID-19 who developed progressive disease had significantly lower baseline ACE levels than those without disease progression. First, the impaired shedding of ACE from inflamed pulmonary vascular endothelium might be a reasonable explanation because respiratory symptoms had been documented in most of our enrolled patients. In addition, more severe inflammation on admission, manifested by significantly higher levels of WBC count, PCT, CRP, ferritin, and overproduction of various proinflammatory cytokines, was observed in the progressive cases, indicating that the entire vascular endothelium might be injured and hence responsible for reduced ACE release. Second, elevated reactive oxygen species (ROS) due to hyperinflammation in COVID-19 has been suggested to have an inhibitory effect on the activity of ACE $[18,19]$. Finally, the overproduction of Ang II documented in SARS-CoV-2-infected patients might lead to a considerable consumption of the ACE pool [20].

More importantly, we further assessed the viral persistence and host responses underlying different levels of ACE, which might assist in better understanding of the role of ACE as a predictor of COVID-19 disease progression. The dynamic detection of viral RNA in clinical specimens revealed that patients with lower ACE level had a significantly higher cumulative detection rate of viral RNA positivity. It is well known that ACE and ACE2 counteract each other to control the metabolism of Ang peptides and play a crucial part in homeostasis [21, 22]. ACEI was widely used to reduce Ang II levels and treat cardiovascular diseases, and is capable of increasing ACE2 mRNA expression in the heart $[21,23]$. Although the regulatory mechanism underlying the switch from ACE to ACE2 is still limited, Yang et al. demonstrated in a cardiac-stressed mouse model that endothelial Brg1-FoxM1 complex could tip the ACE/ACE2 balance to ACE2 expression at the transcription level [24]. Therefore, we speculate that as the critical functional receptor for SARS-CoV-2, ACE2 might be more abundant in patients with COVID-19 with lower ACE levels, resulting in 
an increased susceptibility to SARS-CoV-2 and higher viral load. Furthermore, as shown in our results, an obviously diminished immune response to SARS-CoV-2 infection, as manifested by early significantly lower lymphocyte count, a marked decline in the total T cell, $\mathrm{CD}^{+}{ }^{+} \mathrm{T}$ cell, B cell, and NK cell counts as well as decreased IgG and IgM titers between two detections, was documented for patients with ACE $\leq 33.5 \mathrm{U} / \mathrm{L}$. Such altered immunity was insufficient to eliminate the virus and has been considered a risk factor associated with COVID19 disease progression $[1,25,26]$. In line with our findings, a recent study showed that asymptomatic individuals with a prolonged duration of viral shedding displayed a weaker immune response to SARS-CoV-2 infection than those with symptoms [9]. Therefore, for patients with COVID-19 with lower ACE level, the potential ACE2 accumulation mediated virus entry and the impaired host antiviral immune responses may concomitantly contribute to the delayed virus clearance.

It is well recognized that ACE catalyzes the conversion of Ang I to Ang II, which subsequently exerts its effects including vasoconstriction, blood pressure elevation, activation of pro-inflammatory, and pro-fibrotic pathways via type 1 receptors. This axis was demonstrated to participate in the progression of ARDS and ALI [3]. Thus, theoretically, upregulation of ACE2 levels due to low ACE levels may contribute to vasodilation and alleviation of inflammation. However, we found in the present study that patients with lower ACE levels exhibited overproduction of cytokines as well as higher levels of inflammatory mediators. This may be partly related to a virus-induced cytopathic effect via ACE2 receptors which may play an important role in the development of inflammation. This process is characterized by the activation of the immune system and heavy infiltration of a variety of immune cells and non-immune cells, leading to production of large amounts of proinflammatory cytokines that cause damage to the host [27]. In addition, the hyperinflammation related to lower ACE level might also be attributed to increased serum level of Ang II. ACE2 elevation may enhance susceptibility to SARS-CoV-2. In the case of SARS-CoV-2, the spike protein binds to ACE2 on host cells and is then endocytosed, resulting in the fusion of viral and endosomal membranes, release of the viral genome into the cytoplasm, as well as the reduced and inoperable ACE2 on cells. The process is followed by an increase of serum Ang II and amplification of inflammatory reaction [28]. Moreover, Ang II can be formed through non-ACE pathways. When ACE is blocked, the increased bradykinin could activate the chymase pathway in tissues, allowing production of Ang II, Ang III, and Ang IV [29]. Therefore, the immune-mediated injury during SARS-CoV-2 infection, together with increased Ang II levels due to virus-host interactions and alternative formation pathways might contribute to the hyperinflammation in patients with COVID-19 with lower ACE level.

This study has some limitations. First, the correction of ACE genetic polymorphism is absent in this retrospective study, although the ACE I/D polymorphism showed no association with the poor outcome of pneumonia, including the need for ICU admittance and both inhospital and 28-day mortality [3]. Second, the dynamic changes in serum ACE level as well as its relationship to ACE2 expression in lung tissue were not tested. Last but not least, this cohort size was not large enough to validate the role of ACE in predicting disease progression.

\section{CONCLUSION}

In this retrospective real-world study, we identified that lower level of serum ACE on admission was an independent risk factor for higher incidence of COVID-19 disease progression, which might correlate with prolonged duration of viral shedding, excessive inflammation, and depressed immune response. This study may provide new insights into the clinical management of COVID-19 and enhance the rational allocation of medical resources. 


\section{ACKNOWLEDGEMENTS}

Thank Qin Ning and Weiming Yan for great leadership and contribution to our laboratory. Thank all the participants in this study for their meticulous and patient work. Thank selfless dedications from colleagues in clinical positions in the hospital.

Funding. This study was supported by the Science and Technology Department of Hubei Province under Grant 2020FCA044; Wuhan Science and Technology Bureau under Grant [(2020) 25: 2020020601012228 and 2020020601012236]. The journals Rapid Service Fee was funded by the authors.

Authorship. All named authors meet the International Committee of Medical Journal Editors (ICMJE) criteria for authorship for this article, take responsibility for the integrity of the work as a whole, and have given their approval for this version to be published.

Author Contributions. Weiming Yan and Qin Ning contributed to the study design and had full access to all data in the study. Yuying Chen and Da Huang contributed to data collection, data analysis, literature searches and writing of the manuscript. Wei Yuan, Jiang Chang, Zhize Yuan had roles in data collection. Di Wu, Meifang Han, Xiaoping Luo contributed to review the final version of the manuscript.

Disclosure. Yuying Chen, Da Huang, Wei Yuan, Jiang Chang, Zhize Yuan, Di Wu, Meifang Han, Xiaoping Luo, Qin Ning, and Weiming Yan have nothing to disclose.

Compliance with Ethics Guidelines. The study was performed in accordance with the tenets of the Declaration of Helsinki and the Good Clinical Practice principles. The case series was approved by the Institutional Review Board of Tongji Hospital of Tongji Medical College, Huazhong University of Science and Technology (TJ-C20200101). Written informed consent was waived because of the rapid emergence of this infectious disease.
Data Availability. The datasets used and analyzed during the current study are available from the corresponding author on reasonable request.

Open Access. This article is licensed under a Creative Commons Attribution-NonCommercial 4.0 International License, which permits any non-commercial use, sharing, adaptation, distribution and reproduction in any medium or format, as long as you give appropriate credit to the original author(s) and the source, provide a link to the Creative Commons licence, and indicate if changes were made. The images or other third party material in this article are included in the article's Creative Commons licence, unless indicated otherwise in a credit line to the material. If material is not included in the article's Creative Commons licence and your intended use is not permitted by statutory regulation or exceeds the permitted use, you will need to obtain permission directly from the copyright holder. To view a copy of this licence, visit http://creativecommons.org/licenses/by$\mathrm{nc} / 4.0 /$.

\section{REFERENCES}

1. Chen G, Wu D, Guo W, et al. Clinical and immunological features of severe and moderate coronavirus disease 2019. J Clin Investig. 2020;130: 2620-9.

2. Gaddam RR, Chambers S, Bhatia M. ACE and ACE2 in inflammation: a tale of two enzymes. Inflamm Allergy Drug Targets. 2014;13:224-34.

3. Meijvis SC, Cornips MC, Endeman H, et al. Prognostic value of serum angiotensin-converting enzyme activity for outcome of community-acquired pneumonia. Clin Chem Lab Med. 2011;49: 1525-32.

4. Danilczyk U, Eriksson U, Oudit GY, Penninger JM. Physiological roles of angiotensin-converting enzyme 2. Cell Mol Life Sci. 2004;61:2714-9.

5. Tipnis SR, Hooper NM, Hyde R, Karran E, Christie G, Turner AJ. A human homolog of angiotensinconverting enzyme. Cloning and functional expression as a captopril-insensitive carboxypeptidase. J Biol Chem. 2000;275:33238-43. 
6. Wang Q, Zhang Y, Wu L, et al. Structural and functional basis of SARS-CoV-2 entry by using human ACE2. Cell. 2020;181:894-904.

7. Uçar G, Yildirim Z, Ataol E, Erdoğan Y, Biber C. Serum angiotensin converting enzyme activity in pulmonary diseases: correlation with lung function parameters. Life Sci. 1997;61:1075-82.

8. Zhu Z, Cai T, Fan L, et al. The potential role of serum angiotensin-converting enzyme in coronavirus disease 2019. BMC Infect Dis. 2020;20:883.

9. Long QX, Tang XJ, Shi QL, et al. Clinical and immunological assessment of asymptomatic SARSCoV-2 infections. Nat Med. 2020;26:1200-4.

10. World Health Organization. WHO Coronavirus (COVID-19) Dashboard. Geneva:World Health Organization; 2021 [cited 2021 May 11th]. Available from: https://covid19.who.int/.

11. Chen J, Qi T, Liu L, et al. Clinical progression of patients with COVID-19 in Shanghai, China. J Infect. 2020;80:e1-6.

12. Chen T, Wu D, Chen HL, et al. Clinical characteristics of 113 deceased patients with coronavirus disease 2019: retrospective study. BMJ (Clinical research ed.). 2020;368:m1091.

13. Ruan Q, Yang K, Wang W, Jiang L, Song J. Clinical predictors of mortality due to COVID-19 based on an analysis of data of 150 patients from Wuhan, China. Intensive Care Med. 2020;46:846-8.

14. Zhang X, Yu J, Pan L, Jiang H. ACEI/ARB use and risk of infection or severity or mortality of COVID19: a systematic review and meta-analysis. Pharmacol Res. 2020;158: 104927.

15. Lim J, Cho J, Jeon Y, et al. Adverse impact of reninangiotensin system blockade on the clinical course in hospitalized patients with severe COVID-19: a retrospective cohort study. Sci Rep. 2020;10:20250.

16. Krasowski MD, Savage J, Ehlers A, et al. Ordering of the serum angiotensin-converting enzyme test in patients receiving angiotensin-converting enzyme inhibitor therapy: an avoidable but common error. Chest. 2015;148:1447-53.

17. Mansfield CM, Kimler BF, Henderson SD, Vats TS, Svoboda DJ. Angiotensin-I-converting enzyme in cancer patients. J Clin Oncol. 1984;2:452-6.

18. Michel B, Grima M, Nirina LB, et al. Inhibitory effect of reactive oxygen species on angiotensin I- converting enzyme (kininase II). Clin Exp Pharmacol Physiol. 2001;28:212-8.

19. Saleh J, Peyssonnaux C, Singh KK, Edeas M. Mitochondria and microbiota dysfunction in COVID-19 pathogenesis. Mitochondrion. 2020;54:1-7.

20. Liu Y, Yang Y, Zhang C, et al. Clinical and biochemical indexes from 2019-nCoV infected patients linked to viral loads and lung injury. Sci China Life Sci. 2020;63:364-74.

21. Ocaranza MP, Godoy I, Jalil JE, et al. Enalapril attenuates downregulation of Angiotensin-converting enzyme 2 in the late phase of ventricular dysfunction in myocardial infarcted rat. Hypertension. 2006;48:572-8.

22. Danilczyk U, Eriksson U, Crackower MA, Penninger JM. A story of two ACEs. J Mol Med (Berl). 2003;81: 227-34.

23. Ferrario CM, Jessup J, Chappell MC, et al. Effect of angiotensin-converting enzyme inhibition and angiotensin II receptor blockers on cardiac angiotensin-converting enzyme 2. Circulation. 2005;111: 2605-10.

24. Yang J, Feng X, Zhou Q, et al. Pathological Ace2-toAce enzyme switch in the stressed heart is transcriptionally controlled by the endothelial Brg1FoxM1 complex. Proc Natl Acad Sci USA. 2016;113: E5628-35.

25. Tay MZ, Poh CM, Rénia L, MacAry PA, Ng LFP. The trinity of COVID-19: immunity, inflammation and intervention. Nat Rev Immunol. 2020;20:363-74.

26. Bermejo-Martin JF, Almansa R, Menéndez R, Mendez R, Kelvin DJ, Torres A. Lymphopenic community acquired pneumonia as signature of severe COVID-19 infection. J Infect. 2020;80:e23-4.

27. Khadke S, Ahmed N, Ahmed N, et al. Harnessing the immune system to overcome cytokine storm and reduce viral load in COVID-19: a review of the phases of illness and therapeutic agents. Virol J. 2020;17:154.

28. Hirano T, Murakami M. COVID-19: a new virus, but a familiar receptor and cytokine release syndrome. Immunity. 2020;52:731-3.

29. Cure E, Ilcol TB, Cure Medicine Cumhur. Angiotensin II, III, and IV may be important in the progression of COVID-19. J Renin Angiotensin Aldosterone Syst. 2020:21(4):1470320320972019. 